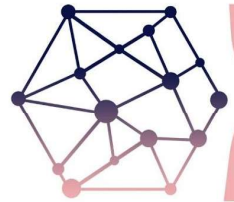 SIBRAGEC

\section{CÁLCULO DO EQUIVALENTE METABÓLICO (MET) NA EXECUÇÃO DO SERVIÇO DE CONTRAPISO ${ }^{1}$}

\author{
GREGOLIS, Caio Dantas (1); PALIARI, José Carlos (2) \\ (1) Universidade Federal de São Carlos, caiodg@gmail.com (2) Universidade Federal de São Carlos, \\ jpaliari@ufscar.br
}

\begin{abstract}
RESUMO
A construção civil é uma indústria tradicional e ainda se mostra conservadora nos métodos de execução de seus serviços, apesar dos avanços tecnológicos. Os trabalhos nos canteiros de obras são realizados, em sua maioria, manualmente, exigindo o emprego de esforços físicos intensos, repetitivos e de longas durações. Os trabalhadores deste setor estão mais sujeitos à fadiga. O objetivo principal deste trabalho é avaliar ergonomicamente a execução do serviço de contrapiso através de uma estimativa do equivalente metabólico, considerando as etapas que englobam desde o recebimento de materiais até a remoção dos resíduos. Para isto, foi utilizado o instrumento de análise ergonômica MET (The Standard Metabolic Equivalent), que estima o dispêndio energético. Foi realizado um estudo de caso, com dados coletados em campo em um empreendimento vertical residencial de alto padrão na cidade de São Paulo, onde foi avaliada a execução de um contrapiso do tipo flutuante. Os resultados obtidos apontaram para uma predominância de atividades de trabalho moderado, exceto para as atividades que envolviam o manejo e transporte de ferramentas e materiais, tendo sido classificadas como trabalho pesado. Estes dados contribuem para a idealização de um compêndio de atividades físicas da construção civil.
\end{abstract}

Palavras chave: Ergonomia, Análise ergonômica, Construção civil, Trabalho, Dispêndio energético.

\begin{abstract}
Civil construction is a traditional industry and is still conservative in the methods of carrying out its services, despite technological advances. Work on construction sites is mostly done manually, requiring the use of intense, repetitive, and long-lasting physical efforts. Workers in this sector are more exposed to fatigue. The main objective of this research is to ergonomically evaluate the performance of the screed service through an estimate of the metabolic equivalent, considering the steps that range from the materials receiving to the waste removal. For this, the ergonomic analysis instrument MET (The Standard Metabolic Equivalent) was used, which estimates the energy expenditure. A case study was carried out, with data collected in the field in a high-end residential vertical building in the city of São Paulo, where the execution of a floating type screed was evaluated. The results obtained pointed to a predominance of moderate work activities, except for the activities that involved the handling and transportation of tools and materials, having been classified as heavy work. These data contribute to the idealization of a compendium of physical activities in civil construction.
\end{abstract}

Keywords: Ergonomics, Ergonomic analysis, Civil construction, Work, Energy expenditure.

\section{INTRODUÇÃO}

A construção civil tem, como uma de suas principais características, a predominância do trabalho manual, isto é, aquele que exige algum grau de esforço físico por parte do trabalhador. Ressalta-se que nem sempre a atividade é realizada da melhor forma, visto

\footnotetext{
${ }_{1}^{1}$ GREGOLIS, C. D.; PALIARI, J. C. Cálculo do equivalente metabólico (MET) na execução do serviço de contrapiso. In: SIMPÓSIO BRASILEIRO DE GESTÃO E ECONOMIA DA CONSTRUÇÃO, 12., 2021, Maceió, Alagoas. Anais[...] Porto Alegre: ANTAC, 2021. p.1-8. Disponível em: https://eventos.antac.org.br/index.php/sibragec/article/view/476. Acesso em: 2 out. 2021.
} 
que este setor é composto, predominantemente, por profissionais de baixa escolaridade, pouca instrução e que aprenderam a realizar suas funções através de ensinamentos passados por pares mais experientes, ou seja, sem um preparo formal.

De acordo com Ray, Parida e Sarkar (2015), a construção civil demanda um grande número de atividades que exigem que o trabalhador as execute manualmente, como elevação, transporte e processamento. Estas atividades podem estar associadas a posturas desconfortáveis, equipamentos e materiais pesados, movimentos repetitivos e condições adversas do meio ambiente e dos postos de trabalho. Todos estes fatores podem contribuir para a fadiga (estresse físico) do trabalhador, o que, por sua vez, são prejudiciais à saúde, promovendo dores e lesões.

Algumas análises ergonômicas com o emprego do instrumento MET (The Standard Metabolic Equivalent) já foram realizadas em atividades da construção civil por Almeida (2019), revestimento de gesso, Carvalho (2016), execução de armaduras, e Bianchini (2015), execução de estruturas e vedações em obras de light steel frame.

O objetivo principal deste trabalho é avaliar ergonomicamente a execução do serviço de contrapiso através de uma estimativa do equivalente metabólico, considerando as etapas que englobam desde o recebimento de materiais até a remoção dos resíduos.

Frente ao exposto, e considerando que há uma lacuna do conhecimento referente ao desenvolvimento de uma análise ergonômica realizada com o auxílio do instrumento MET para a execução do serviço de contrapiso, este trabalho mostra-se contributivo, podendo evidenciar potenciais riscos ergonômicos e, ainda, a possibilidade futura do desenvolvimento de um compêndio de atividades físicas da construção civil. Este trabalho se limita somente à um aspecto da ergonomia física.

\section{REFERENCIAL TEÓRICO}

A ergonomia é uma disciplina de caráter interdisciplinar que contempla a relação entre elementos de um sistema e os seres humanos. Esta relação se dá pelo emprego de teorias, métodos e dados, tendo como principal objetivo melhorar a performance do sistema e do bem-estar do indivíduo (IEA, 2021). Para Iida e Buarque (2016), a adaptação do trabalho ao trabalhador é o que retrata a ergonomia. Isso ocorre através da análise das características dos trabalhadores, a fim de projetar um trabalho que o permita executar de modo que sua saúde não seja comprometida. A ergonomia se divide em: física, cognitiva e organizacional (IEA, 2000).

A análise ergonômica é fruto de uma demanda que surge com o intuito de identificar, avaliar e prevenir riscos ergonômicos relacionados ao trabalho. Para isto, devem ser promovidas minuciosas investigações que forneçam um "raio- $x$ " da situação estudada, considerando a possibilidade de alterações dos produtos e/ou tarefas. Esta análise só é possível com a aplicação de instrumentos de avaliação ergonômica, os quais devem apresentar objetivos definidos e compatíveis com o trabalhado analisado, de modo que seja realizado um contorno da situação, descrevendo-a em detalhes. Posteriormente, problemas são indicados, com ou sem sugestões de melhorias, caso sejam necessárias mais investigações (SIERRA; SANTOS; NICKEL, 2017).

O instrumento de análise ergonômica MET (The Standard Metabolic Equivalent) é responsável por identificar a medida de intensidade de esforço que é exigida de um trabalhador durante a realização de suas funções (RAVAGNANI et al., 2013). Metabolismo basal é a energia necessária somente para manter em funcionamentos as funções vitais do organismo em repouso (IIDA; BUARQUE, 2016).

Segundo a NBR 15575 (ABNT, 2013), o contrapiso, serviço avaliado neste artigo, é definido como: "Estrato com funções de regularizar o substrato, proporcionando uma 
superfície uniforme de apoio, coesa, aderido ou não e adequada à camada de acabamento, podendo eventualmente servir como camada de embutimento, caimento ou declividade".

\section{METODOLOGIA}

\subsection{Estudo de Caso}

Para este trabalho foi adotada a metodologia de estudo de caso, pois permite que seja obtido um amplo e detalhado número de informações sobre o objeto de estudo (GIL, 2002). Yin (2001) considera que os estudos de caso focam em acontecimentos inseridos em contextos da vida real, podendo ter um caráter exploratório, sendo uma forma de investigação empírica.

O principal requisito para a seleção do objeto de estudo era que este fosse um contrapiso executado de modo tradicional (argamassa seca ("farofa") sarrafeada e desempenada).

$\mathrm{O}$ "Estudo de Caso" selecionado compreendeu um empreendimento vertical residencial de alto padrão de 13 pavimentos (1 apartamento por pavimento), localizado na cidade de São Paulo. O contrapiso adotado, do tipo flutuante (não aderido com o substrato), apresentava uma camada de isolamento acústico (manta acústica e lona preta). Foi utilizada argamassa de consistência seca, com pedriscos e macrofibra sintética em seu traço. O serviço foi realizado por dois trabalhadores, sendo um oficial (pedreiro experiente) e um ajudante (pouca experiência). As etapas analisadas compreenderam: abastecimento de materiais, preparação da base, preparação da argamassa, execução da camada de contrapiso e remoção dos resíduos.

A camada de contrapiso foi executada pelo pedreiro enquanto o ajudante foi responsável pelas atividades de preparação da base e apoio (produção, coleta, transporte e despejo de materiais, argamassa e resíduos).

Para cada etapa, foram utilizadas as seguintes ferramentas:

- Abastecimento de Materiais: pá de ferro e girica $\left(0,08 \mathrm{~m}^{3}\right)$, para coleta de areia/pedrisco, e carrinho paleteiro, para a coleta dos sacos de cimento;

- Preparação da Base: talhadeira, vassoura de piaçava, mangueira de nível, colher de pedreiro e trena;

- Preparação da Argamassa: betoneira, masseira, balde, enxada e pá de ferro;

- Execução da Camada de Contrapiso: enxada, soquete, sarrafo, desempenadeiras de madeira e aço, brocha, colher de pedreiro, tábuas, girica $\left(0,08 \mathrm{~m}^{3}\right)$ e pá de ferro;

- Remoção dos Resíduos: vassoura de piaçava, pá de ferro e girica $\left(0,08 \mathrm{~m}^{3}\right)$.

A coleta de dados ocorreu durante a execução do contrapiso do $4^{\circ}$ pavimento, sendo que, para o transporte vertical de materiais e resíduos, foi utilizado um elevador cremalheira. Os materiais estavam armazenados no $2^{\circ}$ subsolo, enquanto os resíduos eram descartados em caçambas localizadas no nível do $1^{\circ}$ subsolo.

A central de produção de argamassa estava localizada no próprio pavimento e era abastecida todas as manhãs e reabastecida ao longo do dia de trabalho. Bem próximo à betoneira estavam os montes de areia/pedrisco, sacos de cimento (normalmente entre $8 \mathrm{e}$ 10 sacos), masseira e 1 saco de macrofibra sintética. $O$ barril com água ficava a uma distância de aproximadamente $7 \mathrm{~m}$.

A distância percorrida pelo ajudante para levar argamassa ao pedreiro variava de acordo com o cômodo onde a camada de contrapiso estava sendo executada. A maior distância identificada estava entre $17 \mathrm{~m}$ e $19 \mathrm{~m}$; entretanto, para fins de cálculo, foram utilizadas as médias. 
Todas as autorizações para a realização do estudo de caso (filmagens, questionários e publicação) foram solicitadas e positivamente atendidas: empresa construtora, engenheiro responsável e trabalhadores responsáveis por executar o serviço de contrapiso.

\subsection{Instrumento de análise ergonômica MET (The Standard Metabolic Equivalent)}

A aplicação do instrumento de análise ergonômica MET (The Standard Metabolic Equivalent) exigiu que os seguintes dados fossem obtidos: códigos de atividade MET [obtidos no Compêndio de Atividades Físicas (CAF), por Farinatti (2003)], peso de um adulto médio (PAM), padronizado em 70kg (ALMEIDA, 2019; BIANCHINI, 2015; COSTA, 2013; FARINATTI, 2003), e duração das tarefas (definida em 1 (uma) hora), ciclos de trabalho e etapas de serviço (em segundos), obtidos por meio da realização de filmagens, coletadas em campo, de todas as etapas de execução do serviço de contrapiso, permitindo a cronometragem. Para a duração dos ciclos e etapas, foram realizadas as médias dos tempos.

Para o cálculo, foi respeitado o seguinte roteiro:

- Duração média do ciclo: (Cm - em segundos);

- Duração média de cada etapa do ciclo: $(\mathrm{Cm}=\mathrm{em} 1+\mathrm{em} 2$... - em segundos);

- Quantidade de ciclos em 1 (uma) hora: $(\mathrm{Qc}=3600$ / Cm);

- Duração total de cada etapa em horas (Et1, Et2 ...): (Et1 = Qc . em1 / 3600);

- Código da atividade MET de cada etapa (MET1, MET2 ...) consultando o CAF;

- Gasto Calórico (Kcal/hora) de cada etapa (D1, D2 ...): (D1 = PAM . MET1 . Et1);

- Gasto calórico total (Kcal/hora) através da somatória do dispêndio energético de cada etapa: $(\mathrm{T}=\mathrm{D} 1+\mathrm{D} 2 \ldots)$;

- Análise do Quadro 1, a seguir, obtido na "NR 15 - Atividades e operações insalubres" (BRASIL, 2014). De acordo com Almeida (2019), como a norma não determina exatamente o limite de troca de um nível para outro, deve-se adotar o limite como sendo a média entre o último valor do nível anterior e o primeiro valor do próximo nível.

Quadro 1 - Taxa metabólica por tipo de atividade

\begin{tabular}{|c|c|}
\hline TIPO DE ATIVIDADE & Kcal/h \\
\hline Sentado em repouso & 100 \\
\hline \multicolumn{2}{|l|}{ TRABALHO LEVE - até $165 \mathrm{Kcal} / \mathrm{h}$} \\
\hline Sentado, movimentos moderados com braços e tronco (ex.: datilografia). & 125 \\
\hline Sentado, movimentos moderados com braços e pernas (ex.: dirigir). & 150 \\
\hline De pé, trabalho leve, em máquina ou bancada, principalmente com os braços. & 150 \\
\hline \multicolumn{2}{|l|}{ TRABALHO MODERADO - de 166 a $370 \mathrm{Kcal} / \mathrm{h}$} \\
\hline Sentado, movimentos vigorosos com braços e pernas. & 180 \\
\hline De pé, trabalho leve em máquina ou bancada, com alguma movimentação. & 175 \\
\hline De pé, trabalho moderado em máquina ou bancado, com alguma movimentação. & 220 \\
\hline Em movimento, trabalho moderado de levantar ou empurrar. & 300 \\
\hline \multicolumn{2}{|l|}{ TRABALHO PESADO - maior que $370 \mathrm{Kcal} / \mathrm{h}$} \\
\hline $\begin{array}{l}\text { Trabalho intermitente de levantar, empurrar ou arrastar pesos (ex.: remoção com } \\
\text { pá). }\end{array}$ & 440 \\
\hline Trabalho fatigante. & 550 \\
\hline
\end{tabular}

Fonte: BRASIL (2014)

Os dados coletados em campo, por meio das filmagens, foram reunidos com os demais dados em planilhas para o cálculo dos dispêndios energéticos. Para cada atividade foi criada uma planilha contemplando as seguintes atividades pelos trabalhadores: buscar manta acústica, buscar pedrisco, buscar areia, buscar cimento, limpeza, colocação da manta acústica, marcação dos níveis, colocação da lona, execução das taliscas, produção 
de argamassa, buscar argamassa, execução da camada de contrapiso e remoção dos resíduos (realizada em duas etapas).

É apresentada no Quadro 2, a seguir, uma planilha de cálculo do dispêndio energético, a título de exemplificação, para a atividade "Preparação da base - Marcação dos Níveis". Para este exemplo, é possível notar que a "Marcação dos Níveis" obteve um valor de dispêndio energético compatível com a classificação de "Trabalho Moderado" (176,57 $\mathrm{Kcal} / \mathrm{h}$ ), de acordo com a classificação apresentada no Quadro 1.

Quadro 2 - Exemplo de cálculo MET - Preparação da Base - Marcação dos Níveis

\begin{tabular}{|c|c|c|c|c|c|c|c|c|}
\hline \multicolumn{9}{|c|}{ MET (The Standard Metabolic Equivalent) - Cálculo do Dispêndio Energético } \\
\hline \multicolumn{9}{|c|}{ Atividade: Preparação da Base - Marcação dos Níveis } \\
\hline Ações & $\begin{array}{c}\text { Duração } \\
\text { Média } \\
\text { Tarefa } \\
\text { (s) }\end{array}$ & $\begin{array}{l}\text { Duração } \\
\text { Média } \\
\text { do Ciclo } \\
\text { (s) }\end{array}$ & $\begin{array}{l}\mathrm{N}^{\circ} \text { de } \\
\text { Ciclos } \\
\text { em } 1 \\
\text { hora }\end{array}$ & $\begin{array}{c}\text { Tempo } \\
\text { Total } \\
\text { da } \\
\text { Tarefa } \\
\text { (h) }\end{array}$ & $\begin{array}{l}\text { Peso } \\
\text { Adulto } \\
\text { Médio } \\
\text { (Kg) }\end{array}$ & MET & $\begin{array}{c}\text { Dispêndio } \\
\text { Energético } \\
\text { (Kcal) }\end{array}$ & $\begin{array}{c}\text { Dispêndio } \\
\text { Energético } \\
\text { (Kcal/h) }\end{array}$ \\
\hline $\begin{array}{c}\text { Buscar e } \\
\text { ajustar }\end{array}$ & \multirow{2}{*}{61} & \multirow{6}{*}{365} & \multirow{6}{*}{9,86} & \multirow{2}{*}{0,17} & \multirow{6}{*}{70,00} & $\begin{array}{l}\text { Código } \\
11.795\end{array}$ & \multirow{2}{*}{35,10} & \multirow{6}{*}{176,57} \\
\hline $\begin{array}{l}\text { mangueira } \\
\text { de nível }\end{array}$ & & & & & & 3 & & \\
\hline $\begin{array}{l}\text { Caminhar } \\
\text { até o ponto }\end{array}$ & \multirow{2}{*}{29} & & & \multirow{2}{*}{0,08} & & $\begin{array}{l}\text { Código } \\
11.795\end{array}$ & \multirow{2}{*}{16,68} & \\
\hline $\begin{array}{c}\text { de } \\
\text { marcação }\end{array}$ & & & & & & 3 & & \\
\hline $\begin{array}{c}\text { Analisar e } \\
\text { marcar } \\
\text { ponto } \\
\end{array}$ & 249 & & & 0,68 & & $\begin{array}{c}\text { Código } \\
11.600 \\
2,3 \\
\end{array}$ & 109,83 & \\
\hline $\begin{array}{l}\text { Guardar } \\
\text { mangueira } \\
\text { de nível }\end{array}$ & 26 & & & 0,07 & & $\begin{array}{c}\text { Código } \\
11.795 \\
3\end{array}$ & 14,96 & \\
\hline
\end{tabular}

Fonte: AUTOR (2021)

\section{RESULTADOS E DISCUSSÕES}

O Quadro 3, a seguir, apresenta um compêndio dos resultados dos dispêndios energéticos obtidos em cada uma das atividades analisadas, apontando quais mostraram-se como "Trabalho Moderado" (em amarelo) e "Trabalho Pesado" (em vermelho). Os valores estão em Kcal/h.

\begin{tabular}{|c|c|c|c|c|}
\hline \multirow{15}{*}{ 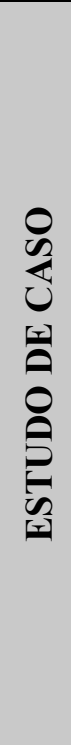 } & \multicolumn{4}{|c|}{ RESULTADOS - MET (The Metabolic Equivalent) - Kcal/h } \\
\hline & \multirow{4}{*}{$\begin{array}{l}\text { Abastecimento de } \\
\text { Materiais }\end{array}$} & Buscar Manta Acústica & 303,39 & Moderado \\
\hline & & Buscar Pedrisco & 393,21 & Pesado \\
\hline & & Buscar Areia & 411,62 & Pesado \\
\hline & & Buscar Cimento & 395,29 & Pesado \\
\hline & \multirow{5}{*}{ Preparação da Base } & Limpeza & 253,36 & Moderado \\
\hline & & Colocação da Manta Acústica & 244,65 & Moderado \\
\hline & & Marcação dos Níveis & 176,57 & Moderado \\
\hline & & Colocação da Lona & 234,52 & Moderado \\
\hline & & Execução das Taliscas & 272,92 & Moderado \\
\hline & Produção da Argamassa & Produção de Argamassa & 327,81 & Moderado \\
\hline & \multirow{2}{*}{$\begin{array}{c}\text { Execução da Camada de } \\
\text { Contrapiso }\end{array}$} & Buscar Argamassa & 502,85 & Pesado \\
\hline & & $\begin{array}{c}\text { Execução da Camada de } \\
\text { Contrapiso }\end{array}$ & 345,12 & Moderado \\
\hline & \multirow{2}{*}{ Remoção dos Resíduos } & Remoção dos Resíduos (1) & 381,47 & Pesado \\
\hline & & Remocão dos Resíduos (2) & 370,25 & Pesado \\
\hline
\end{tabular}

Fonte: AUTOR (2021) 
Dentre as atividades analisadas, predominaram aquelas de trabalho moderado (dispêndio energético entre $166 \mathrm{Kcal} / \mathrm{h}$ e $370 \mathrm{Kcal} / \mathrm{h}$ ), correspondendo a $57,14 \%$ das atividades. As demais se apresentaram como trabalho pesado (dispêndio energético acima de 370 $\mathrm{Kcal} / \mathrm{h}$ ), sendo $42,86 \%$.

Destacaram-se como trabalho pesado as atividades relativas ao "Abastecimento de Materiais" (exceto "Buscar Manta Acústica"), "Remoção dos Resíduos" e "Buscar Argamassa", durante a execução da camada de contrapiso. Estas atividades envolviam como ações principais a utilização da pá de ferro (ferramenta manual pesada) para a coleta de areia, pedrisco, argamassa e resíduos, além da necessidade de elevação e transporte de cargas pesadas como sacos de cimento de $50 \mathrm{~kg}$ e giricas $\left(0,08 \mathrm{~m}^{3}\right)$ totalmente carregadas. Estas ações correspondiam a uma parcela significativa do ciclo.

A Figura 1, a seguir, apresenta as atividades classificadas como trabalho pesado: Coleta e transporte de pedrisco/areia [Figuras 1(a) e 1(b)]; Coleta e transporte de sacos de cimento [Figuras 1(c) e 1(d)]; Coleta e transporte de argamassa [Figuras 1(e) e 1(f)] e Coleta e transporte de resíduos [Figuras $1(\mathrm{~g})$ e 1(h)].

Figura 1 - Atividades classificadas como trabalho pesado

(a)

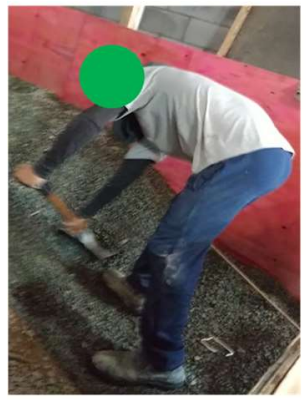

(e)

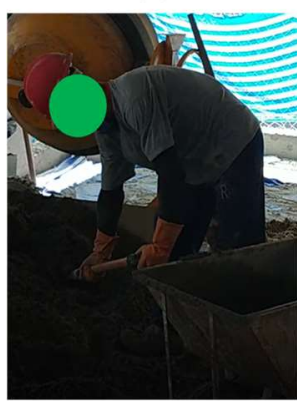

Fonte: AUTOR (2020) (b)

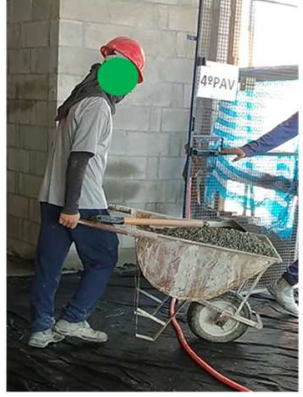

(f)

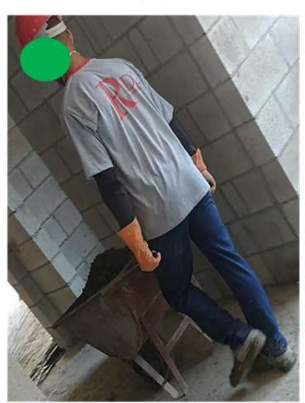

(c)

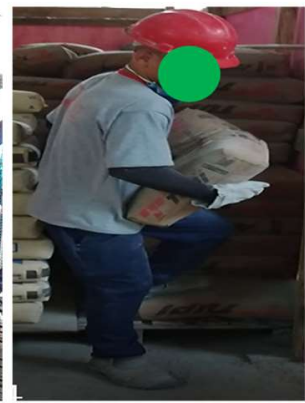

(g)

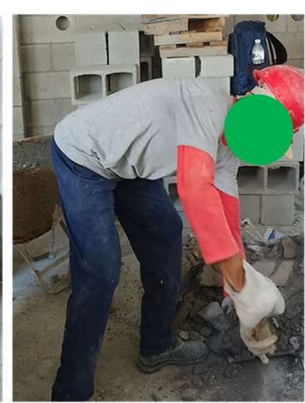

(d)

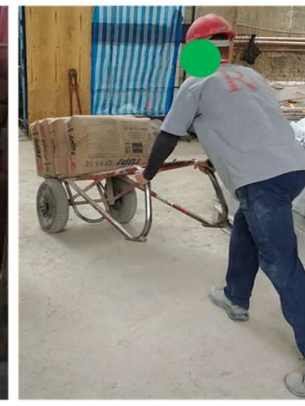

(h)

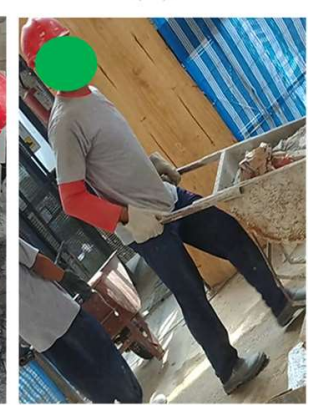

Destacaram-se como trabalho moderado, principalmente, as atividades relativas à "Preparação da Base", "Preparação da Argamassa" e "Execução da Camada de Contrapiso".

Para estas atividades, a utilização de ferramentas pesadas ou o transporte de cargas elevadas não ocupava parcela significativa de tempo, em comparação com a duração total do ciclo. As atividades consideradas como "Trabalho Moderado" eram realizadas entre o pedreiro e o ajudante, enquanto as de "Trabalho Pesado" eram realizadas somente pelo ajudante. As tarefas que ocupavam maior duração de tempo durante um dia de trabalho eram: preparação da argamassa e execução da camada de contrapiso. 


\section{CONSIDERAÇÕES FINAIS}

O domínio de atividades de "Trabalho Moderado" (entre $166 \mathrm{Kcal} / \mathrm{h}$ e $370 \mathrm{Kcal} / \mathrm{h}$ ), $57,14 \%$, pode ser notado pela análise dos resultados obtidos, em contrapartida das atividades de "Trabalho Pesado" (acima de $370 \mathrm{Kcal} / \mathrm{h}$ ), 42,86\%.

Destacam-se:

- A predominância de "Trabalho Moderado" para as atividades relativas à preparação da base, produção da argamassa e execução da camada de contrapiso, realizadas entre o pedreiro e o ajudante;

- A predominância de "Trabalho Pesado" para as atividades relativas ao abastecimento de materiais e remoção dos resíduos, realizadas somente pelo ajudante.

Uma possível explicação para a obtenção destes valores de "Trabalho Pesado" pode estar relacionada à falta de importância e planejamento dada pelas empresas para estas atividades de apoio, visto que não agregam valor direto ao produto final. Vale salientar a merecida importância que deve ser dada ao trabalhador da construção civil, mesmo que este desempenhe uma atividade de pouca ou nenhuma agregação de valor (do ponto de vista teórico), como a coleta e o transporte de materiais e resíduos.

Algumas sugestões de melhorias e boas práticas podem ser citadas:

- Evitar carregar excessivamente o carrinho de mão ou girica, de modo que o trabalhador não precise empreender grande esforço no transporte e despejo de materiais;

- Utilizar sacos de cimento de $25 \mathrm{~kg}$, evitando os de $50 \mathrm{~kg}$. Esta alteração exigiria o emprego de menos esforço por parte do trabalhador e facilitaria o manuseio deste material;

- Caso haja disponibilidade de espaço, manter os sacos de cimento paletizados em pequenos números, de modo que não seja exigido um esforço intenso do trabalhador durante o transporte, e evitando que o mesmo precise coletar e empilhar um saco de cada vez sobre o carrinho paleteiro.

Sugere-se a realização de mais estudos que realizem análises ergonômicas com o emprego do instrumento MET (The Standard Metabolic Equivalent) em outras atividades da construção civil, a fim de promover a informação e criar uma fonte de busca confiável que apresente os gastos calóricos das atividades deste setor, permitindo otimizar as funções de seus trabalhadores, de modo a evitar estresse físico, problemas de saúde e melhorar a qualidade de vida através de melhores condições de trabalho.

\section{AGRADECIMENTOS}

O presente trabalho foi realizado com apoio da Coordenação de Aperfeiçoamento de Pessoal de Nível Superior - Brasil (CAPES) - Código de Financiamento 001.

\section{REFERÊNCIAS}

ABNT. ASSOCIAÇÃO BRASILEIRA DE NORMAS TÉCNICAS. NBR 15575-3: Edificações habitacionais - Desempenho. Rio de Janeiro, 2013.

ALMEIDA, R.B. Análise ergonômica da atividade na execução do serviço de revestimento de gesso. 2019. 217f. Dissertação (Mestrado em Engenharia Civil) Programa de Pós-Graduação em Engenharia Civil da Universidade Federal de São Carlos.

BIANCHINI, G.F. Análise ergonômica do trabalho na execução de estruturas e vedações em obras de light steel frame. 2015. 199f. Dissertação (Mestrado em 
Engenharia Civil) - Programa de Pós-Graduação em Engenharia Civil da Universidade Federal de São Carlos.

BRASIL. Ministério do Trabalho e Emprego. NR 15 - Atividades e operações insalubres. Brasília, DF: Ministério do Trabalho e Emprego, 2014. Disponível em: $<$ https://www.gov.br/trabalho/pt-br/inspecao/seguranca-e-saude-no-trabalho/ctppnrs/norma-regulamentadora-no-15-nr-15> Acesso em: 17 fev. 2021.

CARVALHO, L.C. Análise ergonômica do trabalho na execução de armaduras. 2016. 204f. Dissertação (Mestrado em Engenharia Civil) - Programa de Pós-Graduação em Engenharia Civil da Universidade Federal de São Carlos.

COSTA, S.E.A. Análise ergonômica do trabalho de colheita de citros: comparativo dos métodos de colheita manual e semimecanizado. 2013. 154f. Dissertação (Mestrado em Engenharia de Produção) - Programa de Pós-Graduação em Engenharia de Produção da Universidade Federal de São Carlos.

FARINATTI, P.T.V. Apresentação de uma versão em português do compêndio de atividades físicas: uma contribuição aos pesquisadores e profissionais em fisiologia do exercício. Revista Brasileira de Fisiologia do Exercício, v. 2, n. 2, p. 177-208, Jul. 2003. GIL, A.C. Como elaborar projetos de pesquisa. São Paulo: Editora Atlas, 2002. 176p.

IEA. What is ergonomics? Disponível em: $<$ https://iea.cc/what-is-ergonomics/>. Acesso em: 23 fev. 2021.

IEA. Definição Internacional de ergonomia. Aprovação na Reunião do Conselho Científico da International Ergonomics Association. San Diego, 2000.

IIDA, I.; BUARQUE, L. Ergonomia, projeto e produção. São Paulo: Editora Edgard Blucher, 2016. 359p.

RAVAGNANI, C de F. C; MELO, F. C. L.; RAVAGNANI, F. C. P.; BURINI, F. H. P.; BURINI, R.C. Estimativa do equivalente metabólico (MET) de um protocolo de exercícios físicos baseada na calorimetria indireta. Revista Brasileira de Medicina do Esporte, v. 19, n. 2, p. 134-138, Mar/Abr. 2013.

RAY, P.K.; PARIDA, R.; SARKAR, S. Ergonomic analysis of construction jobs in India: a biomechanical modelling approach. Procedia Manufacturing, v. 3, p. 4606-4612, Jul. 2015 .

SIERRA, I. S.; SANTOS, F. A. N. V.; NICKEL, E. M. Comparativo de usabilidade da ferramenta de avaliação ergonômica Reba (Rapid Entire Body Assessment) nas versões em papel e aplicativo para celular. Revista Gestão e Tecnologia de Projetos, v. 12, n. 2, p. 9-20, Jun. 2017.

YIN, R. K. Estudo de caso: planejamento e métodos. Porto Alegre: Editora Bookman, 2001. 200p. 